\title{
Clinical significance of myocardial involvement in acute idiopathic pericarditis
}

\author{
Aviv Mager, David Berger, Hadas Ofek, Yoav Hammer, \\ Ilana Kedmi, Ran Kornowski \\ Department of Cardiology, Rabin Medical Center - Beilinson Hospital, affiliated to \\ Sackler Faculty of Medicine, Tel Aviv University, Tel Aviv, Israel
}

\begin{abstract}
Background: Acute idiopathic pericarditis (AIP) is frequently accompanied by myocardial involvement (AIPM). Although in acute myocarditis, the myocardial inflammation can lead to life-threatening complications, the outcome of patients with AIPM has been described as good. It remains unclear if a good prognosis of patients with AIPM reflects mild myocardial involvement or good medical management. Methods: A retrospective analysis of life-threatening complications and life-saving interventions in a cohort of 248 consecutive patients admitted to a single medical center between 2006 and 2017 with AIP $(n=169)$ or AIPM $(n=79)$. Major adverse cardiac events $(M A C E)$ included cardiac tamponade, cardiogenic shock, ventricular tachycardia, pericardiocentesis, pericardiectomy, large pericardial effusion and death.

Results: Patients with AIPM were younger than patients with AIP ( $p<0.001)$, and more often had left ventricular dysfunction $(31.6 \% \mathrm{vs} .1 .2 \%, p<0.001)$ and less often had large pericardial effusion (1.3\% vs. $13.6 \%, p=0.002)$, and MACE (5.1\% vs. $14.8 \%, p=0.014)$. Cardiac tamponade occurred in 5.3\% of the patients with AIP as opposed to $1.3 \%$ of the patients with AIPM $(p=0.176)$. Severe left ventricular dysfunction with cardiogenic shock occurred exclusively among patients with AIPM but the rate was low (2.5\%). Life-saving interventions were used in both groups at comparable rates $(2.5 \% \mathrm{vs}$. $5.3 \%, p=0.510)$. There were no in-hospital deaths.

Conclusions: Myocardial involvement in acute pericarditis is associated with a low rate of severe left ventricular dysfunction and cardiogenic shock and a reduced rate of large pericardial effusion, resulting in a lower rate of MACE. Life-saving interventions were used at comparable rates in patients with and without myocardial involvement having excellent survival rates. (Cardiol J 2021; 28, 3: 411-415) Key words: acute idiopathic pericarditis, myocardial involvement, left ventricular dysfunction, tamponade, intervention, outcome
\end{abstract}

\section{Introduction}

Myocardial involvement has been reported in $14.6 \%$ to $60.9 \%$ of patients with acute pericarditis [1-9]. The severity of the myocardial involvement, referred to by some authors as concomitant myocarditis [1], may vary from minimal elevation of a myocardial biomarker level with or without mild left ventricular (LV) dysfunction to severe LV dys- function and ultimately hemodynamic compromise. Although the natural history, characteristics, and outcomes of acute idiopathic pericarditis (AIP) and of acute myocarditis have been studied extensively $[1,10-13]$, the data for acute pericarditis with myocardial involvement (AIPM) are limited [2-6]. In acute myocarditis, myocardial inflammation can lead to life-threatening complications, including severe LV dysfunction, heart failure, cardiogenic

Address for correspondence: Aviv Mager, MD, Department of Cardiology, Rabin Medical Center - Beilinson Hospital, Petach Tikva 4941492, Israel, tel: +972-506844228, fax: +972-39376428, e-mail: a11v12@gmail.com

Received: 24.02 .2019

Accepted: 28.05.2019

This article is available in open access under Creative Common Attribution-Non-Commercial-No Derivatives 4.0 International (CC BY-NC-ND 4.0) license, allowing to download articles and share them with others as long as they credit the authors and the publisher, but without permission to change them in any way or use them commercially. 
shock, ventricular tachycardia and atrioventricular block [10-13]. Recently, an in-hospital rate of death or heart transplant of $3.2 \%$ has been reported [13]. The long-term outcome of patients with acute pericarditis has been described as good, with and without myocardial involvement [3]. However, little is known about the rate of acute phase complications, and it remains unclear whether a good prognosis of patients with AIPM is related to a low rate of severe complications or use of life-saving interventions. Moreover, the finding that the outcome of patients with AIPM is not poorer than patients with acute pericarditis without myocardial involvement is intriguing. The purpose of this study was to examine why myocardial involvement in acute pericarditis is not associated with worse outcomes although it can lead to LV dysfunction. For this purpose, outcomes of patients with acute idiopathic pericarditis were compared with and without myocardial involvement admitted to the documented hospital over an 11-year period and possible differences were examined in acute phase complications and the use of life-saving interventions.

\section{Methods}

The computerized database of the medical center was searched for patients hospitalized between March 1, 2006 and March 1, 2017 with a first episode of AIP or AIPM in whom the diagnosis complied with current guidelines [1]. Patient electronic medical files were retrospectively reviewed for data on mortality, clinical, laboratory and imaging parameters, major adverse cardiac events (MACE), life-saving interventions, and findings on follow-up.

The diagnosis of AIP was based on the presence of at least two of the following criteria: typical chest pain, friction rub, new or worsening pericardial effusion, and typical electrocardiographic changes [1]. Patients in whom a specific etiology for the pericarditis was identified (for example, rheumatoid arthritis, systemic lupus erythematosus, familial Mediterranean fever, tuberculosis, post-myocardial injury syndrome, chronic renal failure, purulent pericarditis, and acute or recent myocardial infarction) were excluded from the study. Patients with active malignancy, pregnant patients, and patients referred from other hospitals were also excluded.

The criteria for the diagnosis of AIPM were the presence of diagnostic criteria for AIP and elevation of cardiac troponin $\mathrm{T}$ (cTnT) level in the presence of a normal serum creatinine level, or an elevated creatine phosphokinase level in the presence of an elevated serum creatinine level.

The size of the pericardial effusion was assessed by echocardiography and categorized semiquantitatively as small, moderate, or large. LV global function and regional wall motion abnormalities were assessed using either echocardiography or cardiac magnetic resonance angiography. Impaired LV function was defined as an abnormal global systolic function or wall motion abnormality. LV systolic function was semi-quantitatively categorized as normal, mildly, moderately or severely decreased. Peak white blood cell count on the first day of admission, peak body temperature, and peak C-reactive protein level were recorded. Exclusion of significant coronary artery disease was done in all patients with myocardial involvement except those at very low risk, using coronary angiography or computed tomographic coronary angiography. The idiopathic nature of the disease was proved by lack of evidence for a specific etiology during hospital stay and on follow-up. Pericardial fluid was analyzed in all the patients when obtained.

The following were categorized as MACE: cardiac tamponade, non-obstructive cardiogenic shock, ventricular tachycardia, large symptomatic pericardial effusion, atrioventricular block and death. The following were recorded as life-saving interventions: pericardiocenthesis or surgical evacuation of pericardial fluid for treatment of cardiac tamponade, cardioversion for sustained ventricular tachycardia, use of intra-aortic balloon counter-pulsation device, use of extracorporeal mechanical oxygenator, and intensive intravenous catecholamine administration with intraarterial pressure monitoring for cardiogenic shock.

The study was approved by the local institutional ethics committee.

\section{Statistical analysis}

Categorical variables are described as numbers and percentages. Continuous variables were evaluated for normal distribution using histograms and $\mathrm{Q}-\mathrm{Q}$ plots and are reported as median and interquartile range. Categorical variables were compared between groups using the $\chi^{2}$ test or the Fisher exact test. Continuous variables were compared using the Kruskal-Wallis test and the Mann-Whitney test. A p value of $<0.05$ was considered significant. All statistical tests were performed using SPSS version 21. 


\section{Results}

The study group included 248 patients $(76 \%$ male) aged $18-89$ years (median, 47 years). Myocardial involvement was found in 79 of them (32\%) and they were categorized as AIPM. Patient baseline characteristics by group are depicted in Table 1 . Compared to the AIP group, the AIPM group was characterized by younger age, lower rate of moderate and large pericardial effusion, higher proportion of LV dysfunction and lower white blood cell count and they were less often given glucocorticoid therapy.

Table 2 shows the rates of in-hospital complications and of use of life-saving interventions. Patients with AIPM had a lower frequency of large symptomatic pericardial effusion. MACE was observed in $30(12.1 \%)$ of the 248 patients. The frequency of MACE was lower in patients with AIPM than in patients with AIP. Severe LV dysfunction and cardiogenic shock occurred only in patients with AIPM but the difference was not statistically significant.

There were no in-hospital deaths. However, life-saving interventions were used in $11(4.4 \%)$ patients: 10 patients underwent evacuation of the pericardial fluid for cardiac tamponade (9 had pericardiocentesis, 1 had surgical evacuation) and 2 patients with cardiogenic shock needed mechanical hemodynamic support (1 extracorporeal oxygenator, 1 intra-aortic balloon counter-pulsation device. One of these patients needed both pericardiocentesis and mechanical support.) Among the
Table 1. Baseline characteristics by group.

\begin{tabular}{|c|c|c|c|}
\hline & $\begin{array}{c}\text { AIP } \\
(n=169)\end{array}$ & $\begin{array}{c}\text { AIPM } \\
(n=79)\end{array}$ & $\mathbf{P}$ \\
\hline Age: & & & $<0.001$ \\
\hline 25 & 39.0 & 26.0 & \\
\hline 50 & 53.0 & 35.0 & \\
\hline 75 & 66.0 & 45.0 & \\
\hline Male gender & $124(73.4)$ & $65(82.3)$ & 0.150 \\
\hline Pericardial effusion: & $118(69.8)$ & $46(58.2)$ & 0.084 \\
\hline None & $49(29.3)$ & $33(41.8)$ & 0.060 \\
\hline Small & $53(31.7)$ & $35(44.3)$ & 0.064 \\
\hline Moderate & $45(36.9)$ & $10(14.9)$ & 0.020 \\
\hline Large & $22(14.9)$ & $1(1.3)$ & 0.002 \\
\hline Moderate or larger & $67(39.6)$ & $11(13.9)$ & $<0.001$ \\
\hline Biomarker elevation & $0(0.0)$ & $79(100.0)$ & $<0.001$ \\
\hline LV dysfunction & $2(11.8)$ & $25(31.6)$ & $<0.001$ \\
\hline CRP: & & & 0.098 \\
\hline 25 & 0.0 & 3.7 & \\
\hline 50 & 11.3 & 7.15 & \\
\hline 75 & 17.2 & 16.7 & \\
\hline WBC: & & & 0.005 \\
\hline 25 & 9090 & 6575 & \\
\hline 50 & 11300 & 8515 & \\
\hline 75 & 13350 & 12775 & \\
\hline Prednisone & $53(31.4)$ & $6(7.6)$ & $<0.001$ \\
\hline
\end{tabular}

Data on age, C-reactive protein (CRP) and white blood cell count (WBC) are medians and percentile 25 and 75 values. All other data are presented as number (percentage). AIP - acute idiopathic pericarditis; AIPM - acute idiopathic pericarditis with myocardial involvement; LV — left ventricular

Table 2. Complications and life-saving interventions by group.

\begin{tabular}{lccc}
\hline & AIP $(\mathbf{n}=\mathbf{1 6 9})$ & AIPM (n = 79) & P \\
\hline Large symptomatic pericardial effusion & $22(14.9)$ & $1(1.3)$ & 0.002 \\
Severe LV dysfunction & $0(0)$ & $2(2.5)$ & 0.102 \\
Cardiogenic shock & $0(0.0)$ & $2(2.5)$ & 0.102 \\
Tamponade & $9(5.3)$ & $1(1.3)$ & 0.176 \\
Pericardial fluid evacuation & $10(5.9)$ & $1(1.3)$ & 0.182 \\
VT/NSVT & $0(0.0)$ & $0(0.0)$ & $>0.999$ \\
Syncope & $2(1.2)$ & $1(1.3)$ & $>0.999$ \\
In-hospital death & $0(0 \%)$ & $0(0 \%)$ & $>0.999$ \\
Major adverse cardiac events & $26(15.4)$ & $4(5.1)$ & 0.002 \\
Life-saving interventions & $9(5.3)$ & $2(2.5)$ & 0.510 \\
\hline
\end{tabular}

Data are presented as number (percentage). AIP — acute idiopathic pericarditis; AIPM — acute idiopathic pericarditis with myocardial involvement; LV — left ventricular; VT — ventricular tachycardia; NSVT — non-sustained ventricular tachycardia 
patients with AIPM, 25 had impaired LV function and thus, met the criteria for perimyocarditis [3]. There was no difference in the rate of life saving interventions in this group, $8 \%$, and the group of patients with AIP $(5.3 \%, \mathrm{p}=0.637)$. Pericardial fluid analysis did not reveal a specific etiology in any of the patients.

\section{Discussion}

The results of this retrospective analysis of consecutive patients with acute idiopathic pericarditis show, for the first time, that acute pericarditis with myocardial involvement is characterized by lower rates of large pericardial effusion and MACE. Severe LV dysfunction and cardiogenic shock may develop in patients with AIPM, necessitating lifethreatening interventions. However, the rate is low and does not significantly increase the rate of MACE. The rates of use of life-saving interventions in patients with and without myocardial involvement are comparable, resulting in a good survival rate for both groups.

Data in the literature regarding the in-hospital course of patients with myocardial involvement are very limited [3-5]. A good survival rate in the present relatively large cohort of patients is in agreement with results of previous reports $[4,5]$. However, previous reports provided only limited information on life-threatening complications and use of life-saving interventions in patients with myocardial involvement. As the current findings show, life-threatening complications occurred in both groups at comparable rates. Because both cardiac tamponade and cardiogenic shock are associated with high mortality rates if untreated, the good outcomes of the present patients with acute pericarditis, with and without myocardial involvement, reflects the success of life-saving interventions rather than a benign natural history.

Although severe LV dysfunction and cardiogenic shock occurred exclusively among patients with AIPM, the proportion of patients with AIPM in need of life-saving interventions in hospital was not higher than in AIP patients. This was explained by the low frequency of this complication, together with a very low rate of cardiac tamponade and need for evacuation of pericardial fluid in the patients with AIPM. The difference in the rate of cardiac tamponade between patients with AIP and AIPM was not statistically significant. However, the significantly lower rates of large and moderate pericardial effusion among patients with AIPM indicate that patients with AIPM are at lower risk of cardiac tamponade. Thus, the lower rate of cardiac tamponade in the present patients with AIPM was probably a true finding.

The absence of atrioventricular block in the present patients was not surprising, as this complication was found to rarely occur in inflammatory cardiac syndromes [3]. Previous reports have demonstrated that myocardial involvement affects younger patients $[2,3,5]$. The younger age of the current patients with AIPM is in agreement with these findings. Interestingly, younger age is typical to acute myocarditis as well [14], supporting the hypothesis that the myocardial involvement in acute pericarditis reflects concomitant myocarditis.

Unlike most previous reports, only patients with idiopathic syndromes were included herein. All patients lived in the same geographic region and acquired the disease during the same period of time. Thus, it is unlikely that our results were biased by shifts in the viral spectrum [15], environmental or genetic factors.

A larger proportion of the patients with AIP received prednisone therapy. Although prednisone therapy is believed to favorably affect the hospital course, MACE occurred more frequently among the patients with AIP. Thus, the less frequent use of prednisone in the current patients with AIPM did not account for the lower rate of MACE in this group.

\section{Conclusions}

Myocardial involvement in patients with acute idiopathic pericarditis is associated with a low rate of severe LV dysfunction and a decreased rate of large pericardial effusion as compared to patients without myocardial involvement. These result in comparable rates of life-threatening complications and of the use of life-saving interventions in patients with acute pericarditis with and without myocardial involvement. Thus, a good prognosis of patients with AIPM despite the occurrence of severe LV dysfunction is explained at least in part by the successful use of life-saving interventions. Because severe LV dysfunction may occasionally occur in AIPM, assessment of LV function and adequate monitoring in these patients are necessary. More research is necessary to clarify whether myocardial involvement is a complication of acute pericarditis or represents a different variant of inflammatory cardiac syndrome characterized by concomitant pericarditis and myocarditis.

Conflict of interest: None declared 


\section{References}

1. Adler Y, Charron P, Imazio M, et al. 2015 ESC Guidelines for the diagnosis and management of pericardial diseases: The Task Force for the Diagnosis and Management of Pericardial Diseases of the European Society of Cardiology (ESC)Endorsed by: The European Association for Cardio-Thoracic Surgery (EACTS). Eur Heart J. 2015; 36(42): 2921-2964, doi: 10.1093/eurheartj/ ehv318, indexed in Pubmed: 26320112.

2. Imazio M, Cecchi E, Demichelis B, et al. Myopericarditis versus viral or idiopathic acute pericarditis. Heart. 2008; 94(4): 498-501, doi: 10.1136/hrt.2006.104067, indexed in Pubmed: 17575329 .

3. Imazio M, Brucato A, Barbieri A, et al. Good prognosis for pericarditis with and without myocardial involvement: results from a multicenter, prospective cohort study. Circulation. 2013; 128(1): 42-49, doi: 10.1161/CIRCULATIONAHA.113.001531, indexed in Pubmed: 23709669.

4. Buiatti A, Merlo M, Pinamonti B, et al. Clinical presentation and long-term follow-up of perimyocarditis. J Cardiovasc Med (Hagerstown). 2013; 14(3): 235-241, doi: 10.2459/ JCM.0b013e328351da6e, indexed in Pubmed: 22644404.

5. Leitman M, Tyomkin V, Peleg E, et al. FACC, FESC. Left ventricular function in acute inflammatory peri-myocardial diseases - new insights and long-term follow-up. Cardiovasc Ultrasound. 2012; 10: 42-48, doi: 10.1186/1476-7120-10-42, indexed in Pubmed: 23121688 .

6. Imazio M, Trinchero R. Myopericarditis: Etiology, management, and prognosis. Int J Cardiol. 2008; 127(1): 17-26, doi: 10.1016/j. ijcard.2007.10.053, indexed in Pubmed: 18221804.

7. Imazio M, Demichelis $\mathrm{B}$, Cecchi $\mathrm{E}$, et al. Cardiac troponin $\mathrm{I}$ in acute pericarditis. J Am Coll Cardiol. 2003; 42(12): 2144-2148, indexed in Pubmed: 14680742.
8. Bonnefoy E, Godon P, Kirkorian G, et al. Serum cardiac troponin I and ST-segment elevation in patients with acute pericarditis. Eur Heart J. 2000; 21(10): 832-836, doi: 10.1053/euhj.1999.1907, indexed in Pubmed: 10781355.

9. Gamaza-Chulián S, León-Jiménez J, Recuerda-Núñez M, et al. Cardiac troponin-T in acute pericarditis. J Cardiovasc Med (Hagerstown). 2014; 15(1): 68-72, doi: 10.2459/ JCM.0b013e3283641161, indexed in Pubmed: 23846679.

10. Imazio M, Gaita F, LeWinter M. Evaluation and treatment of pericarditis. JAMA. 2015; 314(14): 1498-1506, doi: 10.1001/ jama.2015.12763.

11. Caforio ALP, Pankuweit S, Arbustini E, et al. European Society of Cardiology Working Group on Myocardial and Pericardial Diseases. Current state of knowledge on aetiology, diagnosis, management, and therapy of myocarditis: a position statement of the European Society of Cardiology Working Group on Myocardial and Pericardial Diseases. Eur Heart J. 2013; 34(33): 2636-2648, doi: 10.1093/eurheartj/eht210, indexed in Pubmed: 23824828.

12. Sagar S, Liu PP, Cooper LT. Myocarditis. Lancet. 2012; 379(9817): 738-747, doi: 10.1016/S0140-6736(11)60648-X, indexed in Pubmed: 22185868.

13. Ammirati E, Cipriani M, Moro C, et al. Registro Lombardo delle Miocarditi. Clinical Presentation and Outcome in a Contemporary Cohort of Patients With Acute Myocarditis. Circulation. 2018; 138(11): 1088-1099, doi: 10.1161/CIRCULATIONAHA.118.035319, indexed in Pubmed: 29764898.

14. Kytö V, Sipilä J, Rautava P. The effects of gender and age on occurrence of clinically suspected myocarditis in adulthood. Heart. 2013; 99(22): 1681-1684, doi: 10.1136/heartjnl-2013-304449, indexed in Pubmed: 24064227.

15. Shauer A, Gotsman I, Keren A, et al. Acute viral myocarditis: current concepts in diagnosis and treatment. Isr Med Assoc J. 2013; 15(3): 180-185, indexed in Pubmed: 23662385. 